Nonl. Analysis and Differential Equations, Vol. 2, 2014, no. 3, 105 - 115

HIKARI Ltd, www.m-hikari.com

http://dx.doi.org/10.12988/nade.2014.455

\title{
The First Eigenvalue of $p$-Laplacian and Geometric Estimates
}

\author{
Abimbola Abolarinwa \\ Department of Mathematics, University of Sussex \\ Brighton, BN1 9QH, United Kingdom
}

Copyright (c) 2014 Abimbola Abolarinwa. This is an open access article distributed under the Creative Commons Attribution License, which permits unrestricted use, distribution, and reproduction in any medium, provided the original work is properly cited.

\begin{abstract}
We investigate the nonlinear eigenvalue problem for the $p$-Laplacian on compact manifold with zero boundary condition. In particular, we obtain classical estimates of Faber-Krahn inequality and Cheeger-type inequality for the first eigenvalue. As an application we derive Mckeantype estimate and discuss some geometric estimates involving the $L^{p_{-}}$ Sobolev constants.
\end{abstract}

Mathematics Subject Classification: 35P15, 47J10, 53C21

Keywords: $p$-Laplacian, eigenvalue problem, geometric constant

\section{Introduction}

In this paper we study the principal eigenvalue of $p$-Laplacian on Riemannian manifold. Let $M$ be a compact domain in an $n$-dimensional complete, simply connected, Riemannian manifold $\mathcal{M}$ of constant sectional curvature. The $p$ eigenvalue problem for $p \in(1, \infty)$ is the following nonlinear equation

$$
\Delta_{p, g} f(x)=-\lambda|f(x)|^{p-2} f(x), \quad f(x) \neq 0, \quad x \in M
$$

with $f(x)=0$ on $\partial M$. In local coordinates, the $p$-Laplacian is written as

$$
\Delta_{p, g} f(x):=-\frac{1}{\sqrt{|g(x)|}} \sum_{i, j=1} \frac{\partial}{\partial x^{i}}\left(\sqrt{|g(x)|} g^{i j}(x)|\nabla f(x)|^{p-2} \frac{\partial}{\partial x^{j}} f(x)\right),
$$


where $|g|=\operatorname{det}\left(g_{i j}\right)$ and the inverse metric $g^{i j}=\left(g_{i j}\right)^{-1}$. When $p=2, \Delta_{p, g}$ reduces to the usual Laplace-Beltrami operator $\Delta_{2, g} f=-\operatorname{div} \operatorname{grad} f$. It is easily verifiable that the principal symbol of (1.1) is nonnegative everywhere and strictly positive at the neighbourhood of the point where $\nabla f \neq 0$. We know that (1.1) has weak solutions with only partial regularity in general, they are of class $C^{1, \alpha},(0<\alpha<1)$. Notice that the least eigenvalue of compact manifold without boundary or with zero boundary condition is zero with the corresponding eigenfunction being a constant. Hence, we refer to the infimum of the positive eigenvalues as the first (or principal) eigenvalue. The first eigenvalue of $\Delta_{p, g}$ is therefore defined by

$$
\lambda_{1, p}(M)=\inf _{0 \neq f \in W_{0}^{1, p}(M)}\left\{\frac{\int_{M}|\nabla f|_{g}^{p} d \mu_{g}}{\int_{M}|f|_{g}^{p} d \mu_{g}} \mid f \neq 0, \quad f \in W_{0}^{1, p}(M)\right\},
$$

satisfying the following constraint $\int_{M}|f|_{g}^{p-2} f d \mu_{g}=0$. Obviously, the infimum does not change when one replaces $W_{0}^{1, p}(M)$ by $C_{0}^{\infty}(M)$. The corresponding eigenfunction is the energy minimizer of the $p$-Rayleigh quotient and satisfies the following Euler-Lagrange equation

$$
\int_{M}\left[|\nabla f|^{p-2}\langle\nabla f, \nabla \phi\rangle+\lambda|f|^{p-2}\langle f, \phi\rangle\right] d \mu_{g}=0
$$

for $\phi \in C_{0}^{\infty}(M)$ in the sense of distribution. The problem of finding $\lambda_{1, p}$ is related to the problem of finding the best constant $C(M)$ in the $L^{p}$-Sobolev inequality

$$
\|f\|_{L^{p^{*}}} \leq C(M)\|\nabla f\|_{L^{p}}, \quad p^{*}=n p /(n-p),
$$

which is obtained by continuous embedding of $W_{0}^{1, p}(M) \hookrightarrow L^{p}(M)$.

The Laplace-Beltrami operator being the most fundamental second order differential operator in geometric analysis has generated numerous results in literature. This paper therefore aims at reproving some of these classical results (those obtained in the case $p=2$ ) for the case of any $p>1$ and obtain the case $p=2$ as corollaries. Precisely, we obtain the Faber-Krahn inequality, Cheegertype inequality and the Mckean-type estimates. In our proofs we employ the classical ingredients in isoperimetric estimates and the co-area formulas. The books by Chavel [1], P. Li [5] and Schoen-Yau [8] provide details of these results on Laplace-Betrami operator. There are few results for the general case $p \neq 2$, see for instance $[3,4,6,9]$. In section 2 we give definitions of some geometric constants such as Cheeger, isoperimetric and Sobolev constants. We also give the statements of our results. Section 3 is devoted to the proofs of the inequalities and the estimates. In Section 4 we discuss some Sobolev-type inequalities. We show that $L^{1}$-Sobolev inequality implies $L^{p}$-Sobolev inequality for any $p>1$. We use this fact to obtain some useful geometric estimates involving (though, not optimal) Sobolev constant. 


\section{Notation and Statements of results}

Firstly, we recall some notations;

$$
(\operatorname{grad} f)^{i}=(\nabla f)^{i}=g^{i j} \frac{\partial}{\partial x^{j}} f \quad \text { and } \operatorname{div} X=\frac{1}{\sqrt{|g|}} \frac{\partial}{\partial x^{i}}\left(\sqrt{|g|} X^{i}\right),
$$

where $X$ is a smooth vector field. Also we have the metric norm

$$
|\nabla f|_{g}^{2}=g^{i j} \frac{\partial}{\partial x^{i}} f \frac{\partial}{\partial x^{j}} f .
$$

The Riemann structure allows us to define Riemannian volume measure $d \mu_{g}(t)$ on $M$ by

$$
d \mu_{g}=\sqrt{\left|g_{i j}(x)\right|} d x^{i}
$$

In the rest we drop the subscript $g$. We denote by $B_{R}(x)$ a geodesic ball of radius $R$, centred at $x$ in $\mathcal{M}$. The Cheeger, isoperimetric and Soblolev constants on $M$ are denoted by $\mathcal{C}(M), \mathcal{I}(M)$ and $\mathcal{S}(M)$ respectively. The volume of $M$ is denoted by $\operatorname{Vol}(M)$ while $\operatorname{Vol}(\partial M)$ is the $(n-1)$-dimensional volume element on the boundary of $M$. We define a measurable function to be an element of $L^{p}(M)$ if the integral

$$
\|f\|_{p}=\left(\int_{M}|f|^{p}\right)^{\frac{1}{p}}<\infty
$$

holds. We have Hölders inequality for $f \in L^{p}$ and $g \in L^{q}$

$$
\|f g\|_{1}<\|f\|_{p}\|g\|_{q}, \quad 1 / p+1 / q=1, \quad p, q>1 .
$$

The first result we will prove is the following

Theorem 2.1. (Faber-Krahn) Let $M$ be a domain in a complete, simply connected Riemannian manifold $\mathcal{M}$ of constant sectional curvature. Let $B_{R}(x)$ be a geodesic ball of radius $R$ in $M$ such that $\operatorname{Vol}(M)=\operatorname{Vol}\left(B_{R}(x)\right)$. Then the following estimate holds.

$$
\lambda_{1, p}(M) \geq \lambda_{1, p}\left(B_{R}(x)\right) .
$$

There is equality if and only if $M=B_{R}(x)$.

To state the next result we define the Cheeger's isoperimetric constant $\mathcal{C}(M)[2]$ by

$$
\mathcal{C}(M)=\inf _{M_{c}} \frac{\operatorname{Vol}\left(\partial M_{c}\right)}{\operatorname{Vol}\left(M_{c}\right)}, \quad M_{c} \subset \subset M
$$

where the infimum is taken all over manifold $M_{c}$ with compact closure in $M$. Here, $\partial M_{c}$ is assumed to be smooth. 
Theorem 2.2. (Cheeger) Let $M$ be a compact manifold with smooth boundary in a complete Riemannian manifold. Then we have the following estimates

$$
\lambda_{1, p}(M) \geq \frac{1}{p^{p}}(\mathcal{C}(M))^{p} .
$$

Definition 2.3. Define the Sobolev constant $\mathcal{S}(M)$ for $n>1$

$$
\mathcal{S}(M)=\inf _{f \neq 0} \frac{\int_{M}\left(|\nabla f|^{p} d \mu\right)^{n}}{\int_{M}\left(|f|^{\frac{n p}{n-p}} d \mu\right)^{\frac{n-p}{p}}},
$$

where infimum is taken all over compactly supported $C_{0}^{\infty}$ functions in $M$. The $L^{p}$-Sobolev constant holds for $1 \leq p<n$ and as well for all $p \geq n$. Define the isoperimetric constant $\mathcal{I}(M)$ for $n>1$

$$
\mathcal{I}(M)=\inf _{M_{c}} \frac{\operatorname{Vol}\left(\partial M_{c}\right)^{n}}{\operatorname{Vol}\left(M_{c}\right)^{n-1}},
$$

where $M_{c}$ varies over all open submanifold of $M$ with compact closure. Here also, $\partial M_{c}$ is assumed to be smooth.

Theorem 2.4. (Federeer-Fleming) For any $n>p=1$

$$
\mathcal{S}(M)=\mathcal{I}(M) .
$$

The proof can be found in [1, Theorem IV.4] also see [5, Chapter 9] and [8, Chapter III]. As an application of Cheeger-type inequality in Theorem 2.2 we will obtain the following Mckean-type estimates.

Theorem 2.5. (Mckean) Let $\mathcal{M}$ an $n$ dimensional complete (non-compact) simply connected Riemannian manifold of negative constant sectional curvature $-k$. Let $B_{R}(x)$ be a geodesic ball of radius $R$ in $\mathcal{M}$. Then

$$
\lim _{R \rightarrow \infty} \lambda_{1, p}\left(B_{R}(x)\right)=\lambda_{1, p}(M) \geq \frac{1}{p^{p}}((n-1) \sqrt{-k})^{p}
$$

This type of estimate applies only to simply connected complete manifolds with curvature bounded above by negative constant. See Yau [10] for more details.

In our proofs we will use some identities arising from the co-area formula $[1,8]$. Define $M(t)=\{x \in M: f(x)>t\}$ and $A(t)=\{x \in M: f(x)=t\}$, where $t$ is a regular value of $|f|$. We denote by $d A_{t}$ the $(n-1)$-dimensional Riemannian measure of the set $A(t)$. For any $0<f \in C_{0}^{\infty}(M)$ and $1 \leq p \leq \infty$, we have

$$
\int_{M}|\nabla f|^{p} d \mu=\int_{0}^{\infty}\left(\int_{A(t)}|\nabla f|^{p-1} d A_{t}\right) d t
$$


with the following consequences

$$
\begin{gathered}
\operatorname{Vol}(M(t))=\int_{M(t)} d \mu=\int_{0}^{\infty}\left(\int_{A(t)} \frac{1}{|\nabla f|} d A_{t}\right) d t \\
\operatorname{Vol}(\partial M(t))=\int_{M(t)}|\nabla f| d \mu=\int_{0}^{\infty} d A_{t} d t
\end{gathered}
$$

and

$$
\frac{d}{d t} \operatorname{Vol}(M(t))=-\int_{A(t)} \frac{1}{|\nabla f|} d A_{t}
$$

\section{Proofs of results}

\subsection{Proof of Theorem 2.1}

Let $f>0$ be the eigenfunction associated with $\lambda_{1, p}(M)$. Using a symmetrization procedure, we construct a radially decreasing function $f^{*}: B_{R} \rightarrow \mathbb{R}^{+}$. Define the following sets $M_{t}=\{x \in M: f(x)>t\}, A_{t}=\{x \in M: f(x)=t\}$ and $A_{t}^{*}=\left\{x \in B_{R}: f^{*}(x)=t\right\}$ such that $\operatorname{Vol}\left(M_{t}\right)=\operatorname{Vol}\left(B_{R}\right)$ for each $t$. Denote by $d A_{t}$ and $d A_{t}^{*}$, the $(n-1)$-dimensional volume elements of sets $A_{t}$ and $A_{t}^{*}$ respectively. We need to establish that

$$
\lambda_{1, p}(M)=\frac{\int_{M}|\nabla f|^{p} d \mu}{\int_{M}|f|^{p} d \mu} \geq \frac{\int_{B_{R}}\left|\nabla f^{*}\right|^{p} d \mu}{\int_{B_{R}}\left|f^{*}\right|^{p} d \mu}=\lambda_{1, p}\left(B_{R}\right) .
$$

It then suffices to prove

$$
\int_{M}|f|^{p} d \mu=\int_{B_{R}}\left|f^{*}\right|^{p} d \mu \text { and } \int_{M}|\nabla f|^{p} d \mu \geq \int_{B_{R}}\left|\nabla f^{*}\right|^{p} d \mu .
$$

By the co-area formula

$$
\begin{aligned}
\int_{M}|f|^{p} d \mu & =\int_{0}^{\infty} t^{p} \int_{A(t)} \frac{1}{|\nabla f|} d A_{t} d t \\
& =\int_{0}^{\infty} \frac{d}{d t}\left(t^{p}\right) \int_{t}^{\infty} \int_{A(t)} \frac{1}{|\nabla f|} d A_{t} d t=p \int_{0}^{\infty} t^{p-1} \operatorname{Vol}\left(M_{t}\right) d t \\
& =p \int_{0}^{\infty} t^{p-1} \operatorname{Vol}\left(B_{R}\right) d t=\int_{B_{R}}|g|^{p} d \mu .
\end{aligned}
$$

Applying Hölder's inequality we obtain

$$
\int_{A_{t}}|\nabla f|^{p-1} d A_{t} \geq \frac{\left(\int_{A_{t}} d A_{t}\right)^{p}}{\left(\int_{A_{t}}|\nabla f|^{-1} d A_{t}\right)^{p-1}} .
$$


Since $\operatorname{Vol}\left(M_{t}\right)=\operatorname{Vol}\left(B_{R}\right)$ and by $(2.11)$

$$
\int_{\{f=t\}} \frac{1}{|\nabla f|} d t=-\frac{d}{d t} \operatorname{Vol}(M(t)), \quad \int_{\{f=t\}} \frac{1}{\left|\nabla f^{*}\right|} d t=-\frac{d}{d t} \operatorname{Vol}\left(B_{R}\right),
$$

we have

$$
\left(\int_{A_{t}} \frac{1}{|\nabla f|} d t\right)^{\alpha}=\left(\int_{A_{t}^{*}} \frac{1}{\left|\nabla f^{*}\right|} d t\right)^{\alpha}
$$

for any real number $\alpha$. Now using the co-area formula for gradient

$$
\begin{aligned}
\int_{M}|\nabla f|^{p} d \mu & =\int_{0}^{\infty}\left(\int_{A_{t}}|\nabla f|^{p-1} d A_{t}\right) d t \geq \int_{0}^{\infty} \frac{\left(\int_{A_{t}} d A_{t}\right)^{p}}{\left(\int_{A_{t}}|\nabla f|^{-1} d A_{t}\right)^{p-1}} \\
& \geq \int_{0}^{\infty} \frac{\left(\int_{A_{t}^{*}} d A_{t}^{*}\right)^{p}}{\left(\int_{A_{t}^{*}}\left|\nabla f^{*}\right|^{-1} d A_{t}^{*}\right)^{p-1}}=\int_{0}^{\infty}\left(\int_{A_{t}^{*}}\left|\nabla f^{*}\right|^{p-1} d A_{t}^{*}\right) d t \\
& =\int_{B_{R}}\left|\nabla f^{*}\right|^{p} d \mu .
\end{aligned}
$$

This concludes the proof. We only have equality if $M$ is the geodesic ball of radius $R$ in $\mathcal{M}$.

\subsection{Proof of Theorem 2.2}

Let $f(x)>0$ be the corresponding eigenfunction to $\lambda_{1, p}$. Multiplying through the eigenvalue problem $\Delta_{p} f=-\lambda_{1, p}|f|^{p-2} f$ by $f$ and applying integration by parts we have

$$
\lambda_{1, p}(M)=\frac{\int_{M}|\nabla f|^{p} d \mu}{\int_{M}|f|^{p} d \mu} .
$$

Consider for some constant $c(M)$ depending on the geometry of $M$

$$
\int_{M}|\nabla \varphi| d \mu \geq c(M) \int_{M} \varphi, \quad \forall \varphi \in C_{0}^{\infty}(M) .
$$

Suppose $\varphi=f^{p}$, we have by using the Hölder's inequality

$$
\begin{aligned}
\int_{M}\left|\nabla f^{p}\right| d \mu & =p \int_{M}\left|f^{p-1} \nabla f\right| d \mu \\
& \leq p\left(\int_{M}|f|^{p} d \mu\right)^{\frac{p-1}{p}}\left(\int_{M}|\nabla f|^{p} d \mu\right)^{\frac{1}{p}} \\
& =p\left(\int_{M}|f|^{p} d \mu\right)\left(\frac{\int_{M}|\nabla f|^{p} d \mu}{\int_{M}|f|^{p} d \mu}\right)^{\frac{1}{p}} .
\end{aligned}
$$


By this we have

$$
\begin{aligned}
\lambda_{1, p}(M)^{\frac{1}{p}} \int_{M}|f|^{p} d \mu & \geq \int_{M}\left|f^{p-1} \nabla f\right| d \mu=\frac{1}{p} \int_{M}\left|\nabla f^{p}\right| d \mu \\
& =\frac{1}{p} \int_{M}|\nabla \varphi| d \mu \geq \frac{1}{p} c(M) \int_{M} \varphi d \mu \\
& =\frac{1}{p} c(M) \int_{M}|f|^{p} d \mu .
\end{aligned}
$$

Hence

$$
\lambda_{1, p}(M) \geq\left(\frac{1}{p} c(M)\right)^{p}
$$

We now estimate the last inequality from below by applying co-area formula for $\varphi$.

$$
\begin{aligned}
\int_{M}|\nabla \varphi| d \mu & =\int_{-\infty}^{\infty}\left(\int_{A_{t}} d A_{t}\right) d t=\int_{-\infty}^{\infty} \operatorname{Vol}(\partial M(t)) d t \\
& =\int_{-\infty}^{\infty} \frac{\operatorname{Vol}(\partial M(t))}{\operatorname{Vol}(M(t))} \cdot \operatorname{Vol}(M(t)) d t \\
& \geq \inf _{t}\left(\frac{\operatorname{Vol}(\partial M(t))}{\operatorname{Vol}(M(t))}\right) \int_{-\infty}^{\infty} \operatorname{Vol}(M(t)) d t \\
& =\inf _{t}\left(\frac{\operatorname{Vol}(\partial M(t))}{\operatorname{Vol}(M(t))}\right) \int_{M} \varphi d \mu=c(M) \int_{M}|f|^{p} d \mu
\end{aligned}
$$

where the last inequality follows from the definition of $c(M)$. It then follows that

$$
\lambda_{1, p}(M) \geq\left(\frac{1}{p} \mathcal{C}(M)\right)^{p}
$$

\subsection{Proof of Theorem 2.5}

By Cheeger-type inequality (2.3)

$$
\lambda_{1, p}\left(B_{R}(x)\right) \geq \frac{1}{p^{p}}\left(\mathcal{C}\left(B_{R}(x)\right)\right)^{p} .
$$

It suffices to give a lower bound for $\mathcal{C}\left(B_{R}(x)\right)$. Fix a point $y \notin B_{R}(x)$ such that $\rho(y)=d(x, y)$. Since $\mathcal{M}$ is simply connected with negative sectional curvature, $\rho(y)$ is differentiable with $\|\nabla \rho\|=1$. Note that by the Laplacian comparison theorem

$$
\Delta \rho \geq(n-1) \sqrt{-k} \operatorname{coth} \sqrt{-k} \rho \geq(n-1) \sqrt{-k} .
$$


By the co-area formula

$$
\operatorname{Vol}\left(\partial B_{R}(x)\right)=\int_{\partial B_{R}} 1 d A \geq \int_{\partial B_{R}}\langle\nabla \rho, \nu\rangle d A=\int_{B_{R}} \Delta \rho,
$$

where $\nu$ is the outer unit normal along the boundary of $B_{R}(x)$. We have used the fact that $\langle\nabla \rho, \nu\rangle \leq 1$. Therefore

$$
\operatorname{Vol}\left(\partial B_{R}(x)\right) \geq(n-1) \sqrt{-k} \int_{B_{R}} 1 d \mu=(n-1) \sqrt{-k} \operatorname{Vol}\left(B_{R}(x)\right),
$$

which implies that

$$
(n-1) \sqrt{-k} \leq \mathcal{C}\left(B_{R}(x)\right)=\frac{\operatorname{Vol}\left(\partial B_{R}(x)\right)}{\operatorname{Vol}\left(B_{R}(x)\right)}
$$

We conclude that

$$
\lambda_{1, p}\left(B_{R}(x)\right) \geq\left(\frac{(n-1) \sqrt{-k}}{p}\right)^{p} .
$$

The result follows at once.

\section{Geometric estimates on Sobolev constants}

From the definition of the Sobolev constant we define the following $L^{1}$-Sobolev inequality for a function $f \in \mathcal{W}(M)$ and $n>1$

$$
\mathcal{S}(M)^{\frac{1}{n}}\|f\|_{\frac{n}{n-1}} \leq \int_{M}|\nabla f| d \mu
$$

and for any $p>1$

$$
\mathcal{S}(M)^{\frac{1}{n}}\|f\|_{\frac{n p}{n-p}} \leq \int_{M}|\nabla f|^{p} d \mu .
$$

Chavel [1] has proved the following for the case $p=1$.

Theorem 4.1. [1, Theorem IV. 6-7]. If $n=2$, then, $\mathcal{W}(M) \subseteq L^{4}(M)$ and

$$
\int_{M}|\nabla f|^{2} d \mu \geq \frac{\mathcal{S}(M)^{\frac{1}{2}}}{4 \operatorname{Vol}(M)^{\frac{1}{2}}}\|f\|_{4}^{2}
$$

For $n>2, \mathcal{W}(M) \subseteq L^{\frac{2 n}{n-2}}(M)$ and

$$
\int_{M}|\nabla f|^{2} d \mu \geq\left(\frac{\mathcal{S}(M)^{\frac{1}{n}}(n-2)}{2(n-1)}\right)^{2}\|f\|_{\frac{2 n}{n-2}}^{2}
$$

for all $f \in \mathcal{W}(M)$. 
Note that Theorem 2.4 states that the isoperimetric inequality

$$
\operatorname{Vol}(M)^{\frac{n-1}{n}} \leq \mathcal{I}(M) \operatorname{Vol}(\partial M)
$$

is equivalent to the Sobolev inequality for $p=1$ with the same constant. This equivalence implies, by a straightforward approximation, that

$$
\left\|f_{k}\right\|_{\frac{n}{n-1}} \rightarrow \operatorname{Vol}(M)^{\frac{n-1}{n}}
$$

and

$$
\left\|\nabla f_{k}\right\|_{1} \rightarrow \operatorname{Vol}(\partial M) .
$$

1 Our first remark is to show that the Sobolev inequality in the case $p=1$ implies those of the case $p>1$. This has been done for Euclidean case in [7]. Consider the $L^{1}$ - Sobolev inequality

$$
\mathcal{S}(M)^{\frac{1}{n}}\|f\|_{\frac{n}{n-1}} \leq \int_{M}|\nabla f| d \mu, \quad f \in C_{0}^{\infty}(M) .
$$

Fix $p>1$. For any $\alpha:=(n-1) p /(n-p)>1$, noting that $|f|^{\alpha}$ is $C^{1}$ with compact support, we have

$$
\begin{aligned}
\int_{M} \nabla|f|^{\frac{(n-1) p}{n-p}} & =\frac{p(n-1)}{n-p} \int_{M}|f|^{\frac{(n-1) p}{n-p}-1}|\nabla f| \\
& \leq \frac{p(n-1)}{n-p}\left(\int_{M}|f|^{\left(\frac{(n-1) p}{n-p}-1\right) q} d \mu\right)^{\frac{1}{q}}\left(\int_{M}|\nabla f|^{p} d \mu\right)^{\frac{1}{p}},
\end{aligned}
$$

where we have used Hölder's inequality with $1 / p+1 / q=1$. Therefore

$$
\|f\|_{\frac{\alpha n}{n-1}}^{\alpha} \leq \frac{p(n-1)}{(n-p) \mathcal{S}(M)^{\frac{1}{n}}}\left(|f|^{\frac{n p}{n-p}} d \mu\right)^{\frac{p-1}{p}}\left(\int_{M}|\nabla f|^{p} d \mu\right)^{\frac{1}{p}},
$$

which implies

$$
\|f\|_{\frac{n p}{n-p}}^{\frac{(n-1) p}{n-p}} \leq \frac{p(n-1)}{(n-p) \mathcal{S}(M)^{\frac{1}{n}}}\|f\|_{n p /(n-p)}^{n(p-1) /(n-p)}\|\nabla f\|_{p} .
$$

Therefore

$$
\|f\|_{\frac{n p}{n-p}}^{p} \leq\left(\frac{p(n-1)}{(n-p) \mathcal{S}(M)^{\frac{1}{n}}}\right)^{p} \int_{M}|\nabla f|^{p} .
$$

Thus, we have proved

\footnotetext{
${ }^{1}$ The isoperimetric inequality in $\mathbb{R}^{n}$ asserts that among sets having a smooth boundary of a given finite $(n-1)$-dimensional measure, the ball has the largest $n$-dimensional volume.
} 
Proposition 4.2. For any $n \geq 2,1 \leq p<n$, then $f \in L^{\frac{n p}{n-p}}$ and

$$
\|f\|_{\frac{n p}{n-p}} \leq \frac{p(n-1)}{(n-p) \mathcal{S}(M)^{\frac{1}{n}}}\|\nabla f\|_{p} .
$$

This result also holds for all $p>n$.

The second useful remark we make is that if $L^{p}$-Sobolev inequality holds on $M$, then, $L^{q}$-Sobolev inequality also holds for all $p \leq q<n$. This also demonstrates that the strength of a Sobolev inequality decrease as $p$-increases. We follow the previous calculation closely (see also [1] and [7]). Now denote

$$
C=C(M, n, p):=\frac{p(n-1)}{(n-p) \mathcal{S}(M)^{\frac{1}{n}}} .
$$

Then by the last proposition, the $L^{p}$-Sobolev inequality reads

$$
\|f\|_{\frac{n p}{n-p}} \leq C\|\nabla f\|_{p}
$$

In a similar way as before we apply the last inequality to $|f|^{\alpha}$, where $\alpha$ will be chosen as $\alpha=\frac{q(n-p)}{p(n-q)}>1$, we then have by Hölder's inequality

$$
\begin{aligned}
\|f\|_{\frac{\alpha n p}{n-p}}^{\alpha} & \leq \alpha C\left(\int_{M}|f|^{\alpha-1}|\nabla f|^{p} d \mu\right)^{\frac{1}{p}} \\
& \leq \alpha C\left(\int_{M}|f|^{p(\alpha-1) \cdot q /(q-p)} d \mu\right)^{\frac{1}{p} \frac{q-p}{q}}\left(\int_{M}|\nabla f|^{p \cdot \frac{q}{p}} d \mu\right)^{\frac{1}{p} \cdot \frac{p}{q}} .
\end{aligned}
$$

Choosing $\alpha$ as indicated above we obtain

$$
\begin{gathered}
\frac{p q(\alpha-1)}{q-p}=\frac{n q}{n-q}=\frac{\alpha n p}{n-p}, \quad \alpha-1=\frac{n(q-p)}{p(n-q)} \\
\text { and } \quad \frac{q(n-p)}{p(n-q)}-\frac{n(q-p)}{p(n-q)}=1 .
\end{gathered}
$$

Therefore

$$
\|f\|_{\frac{n q}{n-q}}^{\frac{q(n-p)}{p(n-q)}} \leq \frac{q(n-p)}{p(n-q)} C\left(\int_{M}|f|^{n q /(n-q)} d \mu\right)^{\frac{q-p}{p q}}\left(\int_{M}|\nabla f|^{q} d \mu\right)^{\frac{1}{q}} .
$$

Then we arrive at

$$
\|f\|_{\frac{n q}{n-q}} \leq \frac{q(n-1)}{(n-q) \mathcal{S}(M)^{\frac{1}{n}}}\|\nabla f\|_{q}
$$

Acknowledgements. The author wishes to thank the anonymous referees. His research is supported partly by the University of Sussex and by Nigeria FGN/TETfund. 


\section{References}

[1] I. Chavel, Eigenvalues in Riemannian Geometry, Academic Press, Inc. NY, (1984).

[2] J. Cheeger, A lower bound for the smallest eigenvalue of the Laplacian, Problems in Analysis, Princeton Univ. Press, N. J. (1970), 195-199.

[3] S. Kawai, N. Nakauchi, The first eigenvalue of the $p$-Laplacian on a compact manifold, Nonlinear Analysis, 55 (2003), 33-46.

[4] I. Ly, The first eigenvalue for the $p$-Laplacian operator, J. Ineq. Pure and Appl. Math, 6(3), (2005), 1-12.

[5] P. Li, Geometric Analysis, Geometric Studies in Advanced Mathematics, Cambridge University Press (2012).

[6] B. P. Lima, J. F. Montenegro, N. L. Santos, Eigenvalues estimates for the first eigenvalue of the $p$-Laplace operator on manifolds, arXiv:0808.2028 [math.DG] 14 Aug 2008.

[7] L. Saloff-Coste Aspects of Sobolev-Type Inequalities, Cambridge University Press (2002).

[8] R. Schoen, S.-T. Yau, Lectures on differential geometry, International Press, Cambridge, MA, 1994.

[9] H. Takeuchi, On the first eigenvalue of the $p$-Laplacian in a Riemannian manifold, Tokyo J. Math 21(1)(1998), 136-140.

[10] S.T. Yau, Isoperimetric constant and the first eigenvalue of a compact manifold, Ann. Sci Ec. Norm. Super, Paris 487,(1975), 487-507.

Received: May 17, 2014 